\begin{tabular}{|c|c|c|c|c|c|}
\hline Cuadernos I. Geográfica & 15 & $1-2$ & $67-74$ & Logroño & 1989 \\
\hline
\end{tabular}

\title{
LOS CONGLOMERADOS SUELTOS TERCIARIOS DEL BORDE NORTE DE LA SIERRA DE CAMEROS
}

\author{
Félix Pérez-Lorente* \\ Sara Pavía Santamaría** \\ Carlos Pereda Olasolo**
}

\begin{abstract}
RESUMEN
Se examinan, en este trabajo, la edad y situación estratigráfica de los conglomerados siliceos no cementados del Norte de la Sierra de Cameros. Tales rocas son de tres edades distintas y aún no se ha encontrado ninguna característica litológica o textural que permita separarlas: las inferiores son del Oligoceno Superior (Chantiense o más antiguas); las medias son de edad Vindoboniense; y las más modernas del Pontiense. Los conglomerados inferiores y medios son anteriores a la fase de cabalgamiento principal de la zona; los superiores posteriores a ella.
\end{abstract}

\section{SUMMARY}

The present paper is a study of the age of the siliceous conglomerates not consolidates situated in the Northern Sierra de Cameros. The age of these rocks are: Upper Oligocene (Chattian or older) the oldest; middle Miocene (Vindobonian) the intermediate; and Upper Miocene (Pontian) the youngest. While the middle and lower conglomerates are affected by alpine overthrust, the upper ones are not.

PALABRAS CLAVES: conglomerados, Sierra de Cameros, Terciario KEY WORDS: conglomerates, Sierra de Cameros, Tertiary 


\section{PEREZ, PAVIA Y PEREDA}

\section{INTRODUCCION}

Los conglomerados sueltos del borde Norte de las Sierras de la Demanda y Cameros han sido objeto de discusión con respecto a su edad, su génesis y su posición estratigráfica. $\mathrm{Al}$ cartografiar dicho borde norte con objeto de estudiar las características tectónicas del mismo, han surgido datos que hacen modificar en parte los esquemas propuestos hasta ahora.

SANCHEZ LOZANO (1894) los atribuyó al Cuaternario, en lo que parece ser la primera referencia escrita. SCHRIEL (1930) dijo que pueden ser del Terciario Superior, del Plioceno o incluso del Cuaternario. En la Hoja de Calahorra de 1947 se dice los conglomerados sueltos de esta hoja y alrededores son Miocenos.

RIBA (1955) encuentra la dicotomía entre el sector de Agueda-Arnedo y Arnedo-Belorado. En la primera, sobre una superficie de erosión descansan los conglomerados cuya edad es Vindoboniense-Pontiense; en la segunda solamente se puede decir que serian del Mioceno Superior. Según este autor, la sedimentación de los conglomerados terminaría en el Pontiense por lo que la edad en la que están todos incluidos va desde el Vindoboniense al final del Mioceno.

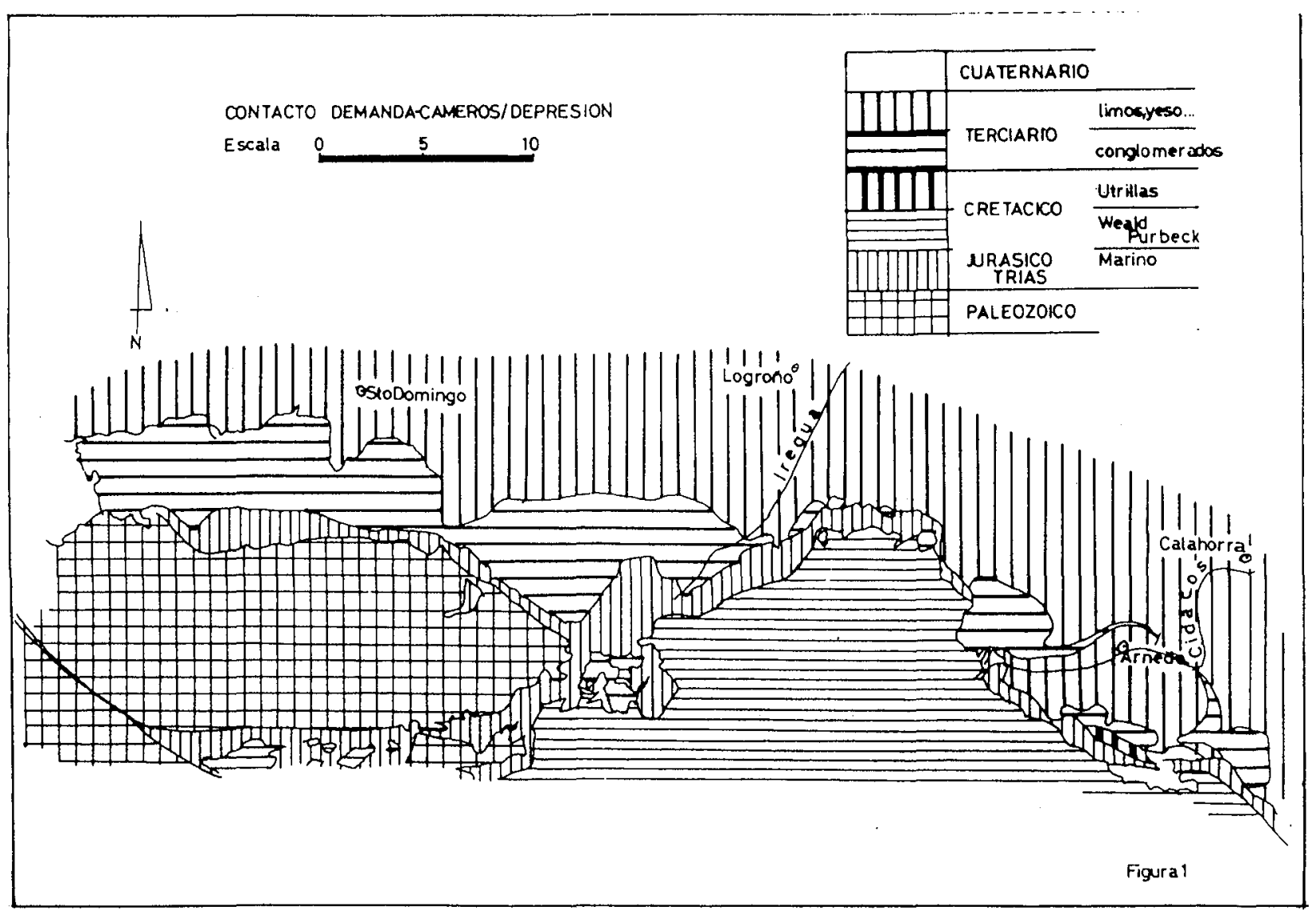

En la hoja 1:200.000 de Logroño (1971) los conglomerados sueltos tienen edades que abarcan todo el Oligoceno y Mioceno, aunque se hace alguna distinción de afloramientos. En las Hojas 1:50.000 del Plan Magna se dan las edades siguientes: Hoja de Logroño (1975) Arquitaniense-Vindoboniense; Hoja de Calahorra (1977) edad de Chatiense-Aquitaniense a Pontiense; Hoja de Cervera del Río Alhama (1982) Pontiense. 


\section{CONGLOMERADOS TERCIARIOS}

CUENCA $(1983,1985)$ data paleontológicamente los niveles de Autol en el Mioceno Inferior, por lo que algunas formaciones de conglomerados sueltos quedan por debajo de esta edad. DAAMS y VAN DER MEULEN (1984) sitúan los yacimientos de Autol en el Chatiense por lo que se confirma la edad oligocena de estos conglomerados (ORTEGA y PEREZLORENTE, 1984).

\section{SITUACION GEOGRAFICA Y GEOLOGICA}

Estos conglomerados sueltos se encuentran en el borde Norte de la Sierra de Cameros. Parece que afloran siguiendo una banda que desde Oeste a Este iría desde la terminación de la Sierra de la Demanda en Burgos hasta, al menos, las estribaciones del borde Norte del Moncayo. En este trabajo se estudia (figura 2) el sector comprendido entre los ríos Iregua y Cidacos.

La edad litológica de las rocas que afloran en La Rioja permiten que en ella se diferencien varios dominios claros. El borde norte de la Sierra de Cameros es uno de ellos (figura 1). Al sur de esta línea está el conjunto Mesozoico constituido por:

a) las rocas de facies continental del Jurásico y Cretácico, tradicionalmente aquí de facies Weald (cuarcitas, grauvacas, limolitas o pizarra y calizas)

b) por las rocas marinas del Jurásico y las facies continentales del Trías germánico

c) las rocas detríticas sueltas de facies Utrillas.

Al norte de la línea, formaciones continentales del Terciario (Oligoceno y Mioceno) casi exclusivamente conglomeráticos en las proximidades de este borde, con intercalaciones menores de arenas y limos.

Los conglomerados que nos ocupan se han definido como «...de composición poligénica, dominantemente silícea, poco o sin apenas cemento...» (RIBA, 1964) y también «...no cementados, de matriz generalmente arcillosa o limosa... hetrométricos...» (ORTEGA y PEREZ-LORENTE, 1984), y se encuentran a ambos lados de la línea que separa los dominios.

Los conglomerados cementados que los acompañan se han definido así «...conglomerados calcáreos masivos y bien cementados...» (RIBA, 1964); "...conglomerados hetrométricos con abundantes cantos de caliza... generalmente masivos...) (ORTEGA y PEREZ-LORENTE, 1984). El cemento de estos conglomerados es calizo.

En la cartografia de la Figura 1 no se han separado los distintos tipos de conglomerado.

$\mathrm{Al}$ norte, y en parte subyacentes bajo ellas, se encuentran las formaciones detríticas más finas del terciario y las rocas de precipitación química. Al sur, y también en parte subyacentes, las de las unidades mesozoicas cabalgantes

conglomerados sueltos discordancia

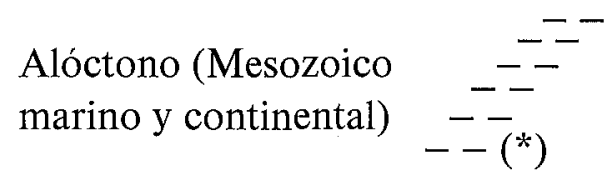

Autóctono (Terciario

del Valle del Ebro)

(*) Los trazos indican contacto cabalgante

$(* *)$ También tiene conglomerados sueltos en paquetes cartografiables a escala 1.50 .000 . 


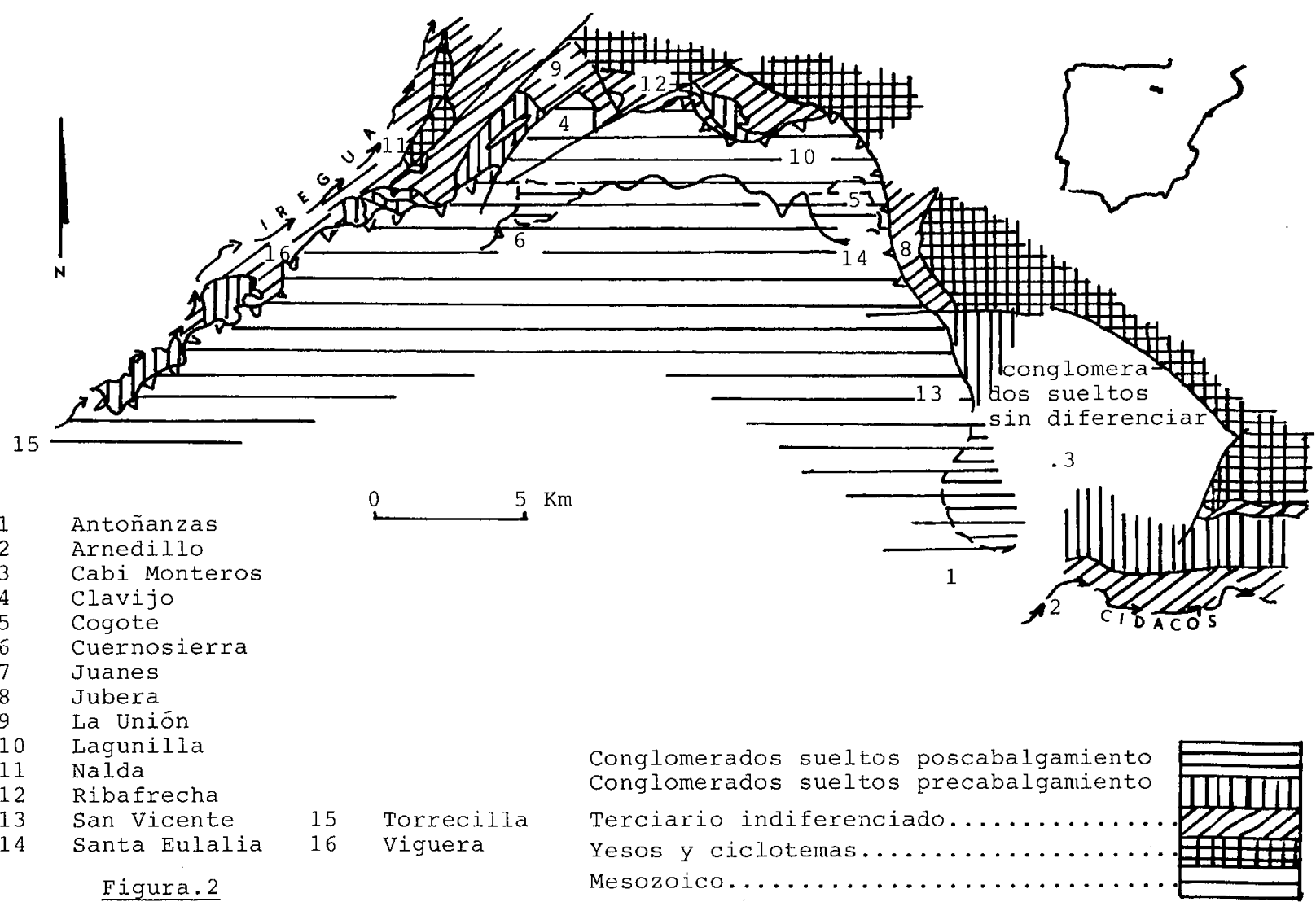

\section{LOS CONGLOMERADOS SUELTOS}

Se sitúan siguiendo el contacto cabalgante entre los Cameros y la Depresión del Ebro. Todos los conglomerados sueltos tienen la composición que se indica: o no hay, o son muy escasos los cantos de caliza; abundan por zonas los de composición grauváquica o cuarcítica. Los cantos más silíceos proceden de áreas próximas del sur (las facies Weald); la matriz, cuando se ve, es arenosa o arcillosa, y parece que hay capas delgadas de arcillas y arenas. El color de la arcilla es rojo en muchos afloramientos; el color de la arena parece que es amarillento a gris verdoso. En general los colores que se ven van desde los pardorrojizos al gris.

La forma de los cantos suele ser poco esférica, aunque muy redondeada. No se han visto estructuras superficiales de los cantos (estrías, etc.)

La mayor parte de los afloramientos da morfología redondeada, de vertiente estable, debido a que los cantos se sueltan con facilidad y no dan paredes verticales o rectas. Sobre ellos no se forman ni glacis ni las superficies de erosión tan típicas del resto de la Depresión, debido probablemente a la composición y calibre de los cantos del conglomerado.

Como los cantos se desprenden con facilidad, tanto sus contactos como su estructura permanecen invisibles. Por otra parte hay conglomerados sueltos discordantes sobre rocas infrayacentes que también tienen grandes niveles de conglomerados sueltos (pero más antiguos) como en Cabi Monteros y Juanes, es decir, conglomerados sueltos miocenos sobre otros oligocenos. Hay contacto estratigráfico concordante entre conglomerados sueltos y 


\section{CONGLOMERADOS TERCIARIOS}

otras rocas al Norte de Santa Eulalia, al Norte de Arnedillo, al Noreste de Jubera. Al Norte de Antoñanzas, al Este de San Vicente de Robres y Valtrujal, y al Norte de Trevijano los conglomerados sueltos descansan sobre rocas jurásicas, cretácicas o triásicas discordantemente.

Aunque no se ha medido el calibre de los cantos, la mayor abundancia de cantos pequeños, (menos de $40 \mathrm{~cm}$.) se encuentran en los afloramientos de Juanes, en los de cerca de Ribafrecha, y en los afloramientos del Iregua. En Cabi Monteros hay de todo. Aparentemente los cantos mayores abundan en la parte más alta de algunos afloramientos (Juanes, Cabi Monteros y en las manchas de Cuernosierra y Cogote). Aquí hay cantos de más de un metro de diámetro (en Cuernosierra se ha medido uno de 2’30 metros).

Como se viene diciendo existen varias formaciones de conglomerados sueltos. Todas ellas deben ser terciarias, aunque una de ellas es posterior a todas las demás y no está interestratificada con el resto de los materiales terciarios.

Esta diferenciación nos implica que al menos hay dos formaciones de conglomerados sueltos, aunque hay casos de interpretación difícil a la hora de asignar a uno u otro paquete los conglomerados sueltos como en los afloramientos de Cabi Monteros y Juanes.

\section{ESTRATIGRAFIA}

Se describen cortes sobre hechos sobre el terreno, teniendo en cuenta datos de cartografía geológica

Al Norte de Torrecilla siguiendo el curso del Iregua

muro - más de $150 \mathrm{~m}$. de conglomerados sueltos

- $100 \mathrm{~m}$. de conglomerados cementados

- $140 \mathrm{~m}$. de conglomerados sueltos

El sistema de fallas que sigue impide ver la sucesión por lo que se reinicia el perfil desde Viguera hasta Nalda

muro. - más de $130 \mathrm{~m}$. de conglomerados cementados

- $60 \mathrm{~m}$. de conglomerados sueltos que se acuñan hacia el Norte

- $100 \mathrm{~m}$. de conglomerados cementados

- más de $150 \mathrm{~m}$. de conglomerados sueltos

- $80 \mathrm{~m}$. de conglomerados cementados

- $60 \mathrm{~m}$. de ciclotemas costituidos por la repetición de la secuencia: conglomerados; arenas; limos y arcillas; carbón (visto solamente en un punto) y; calizas. Estos ciclotemas pasan hacia el Norte a alternancias de limos y margas o calizas. En este paquete hay al menos un nivel de yeso.

- más de $70 \mathrm{~m}$. de areniscas y limos rojizos

La sucesión que se encuentra en Juanes es la siguiente: 


\section{PEREZ, PAVIA Y PEREDA}

muro. - $200 \mathrm{~m}$. de conglomerados sueltos

- menos de $50 \mathrm{~m}$. de conglomerados cementados

- más de $20 \mathrm{~m}$. de conglomerados sueltos de calibre grande

Desde la Unión a Clavijo se sigue.

muro. - yesos

- $80 \mathrm{~m}$. de limos y areniscas rojizas

- más de $90 \mathrm{~m}$. de conglomerados cementados

La sucesión al Norte de Santa Eulalia es:

muro. - alternancias rojizas con conglomerados cementados de la potente formación de Arnedo.

- más de $600 \mathrm{~m}$. de conglomerados sueltos. Parte de estos conglomerados yacen bajo los conglomerados de Quel (ORTEGA y PEREZ-LORENTE, 1984)

Al este de Jubera encontramos la siguiente sucesión:

muro. - más de $80 \mathrm{~m}$. de areniscas y limos rojizos

- $100 \mathrm{~m}$. de alternancias de areniscas y limos con calizas

- los conglomerados sueltos de Cabi Monteros.

Al sintetizar todos estos datos, teniendo en cuenta la continuidad lateral de algunas capas, vemos que se puede establecer la siguiente sucesión general:

muro. - más de $600 \mathrm{~m}$. de alternancias de conglomerados sueltos y cementados. Relativa proximidad al borde de la cuenca (cortes de Torrecilla y de Viguera). En algunos puntos más alejados del borde (Jubera, Santa Eulalia) predominan las alternancias de areniscas y limos rojizos.

- capas con calizas y yesos (Nalda, La Unión, Jubera, Santa Eulalia). Parece que en esta posición hay un retroceso del borde de la cuenca porque no se encuentra la transición hacia facies más proximales.

- más de $250 \mathrm{~m}$. de alternancias de conglomerados sueltos y cementados (Juanes y posiblemente una gran parte de los qué afloran en Cabi Monteros).

techo.- desconocido. Tras el intervalo en el que terminó el cabalgamiento de las sierras o al menos el último impulso fuerte

- la formación terminal de conglomerados sueltos, discordante.

No se separan los conglomerados sueltos de los cementados de la parte inferior de la sucesión porque se ven indentaciones de los unos en los otros.

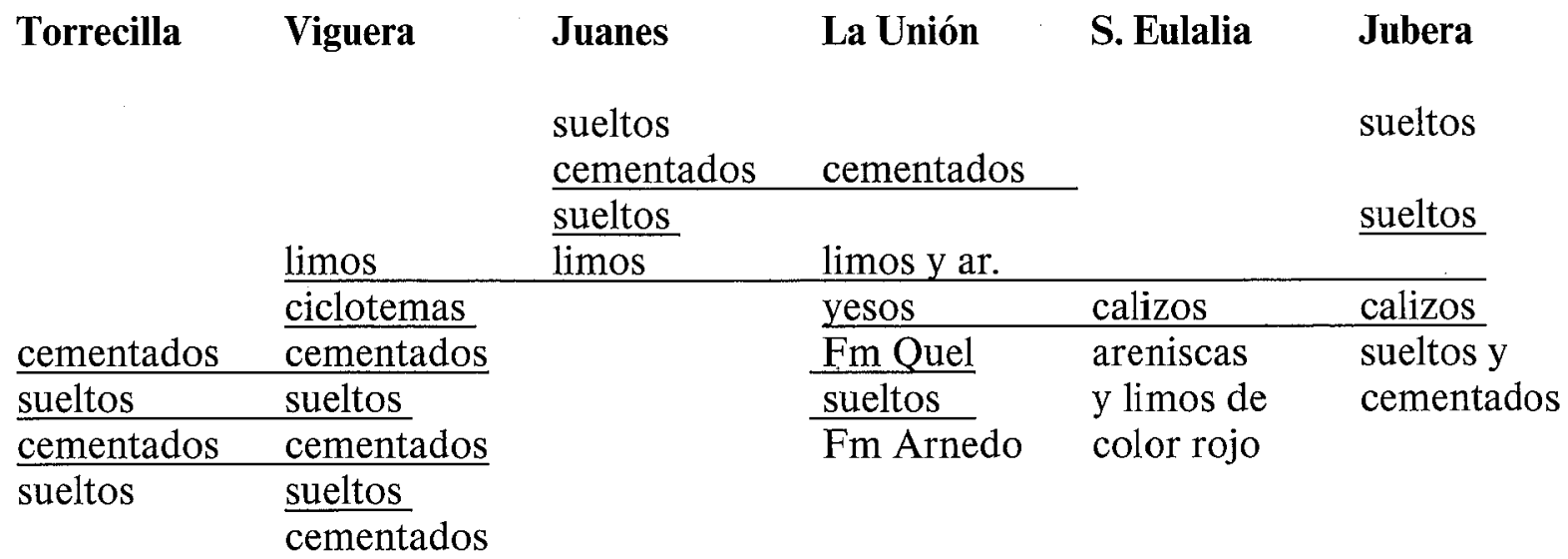




\section{CONGLOMERADOS TERCIARIOS}

\section{DISCUSIONES Y CONCLUSIONES}

Los conglomerados anteriores a los cabalgamientos están relacionados con la tectónica puesto que se encuentran alineados siguiendo un borde activo. Los más de $600 \mathrm{~m}$. de potencia de conglomerados en capas alternantes (cementados y no) implican un movimiento de subsidencia mayor. Como son diferentes: el calibre, la naturaleza y el cemento que traba a los cantos, este depósito restringido a una banda (de los conglomerados) no se debe a efectos climáticos o ambientales. Los cambios de facies se producen con tal rapidez que el área fuente tenía que estar muy próxima al borde activo.

Para justificar la ausencia o presencia de cemento, así como la naturaleza de los cantos mayoritarios, se ha recurrido hasta ahora a condicionamientos climáticos (SANCHEZ LOZANO, 1984; ORTEGA y PEREZ-LORENTE, 1984) o tectónicos (RIBA, 1955). E1 segundo caso queda descartado dado que no se puede suponer erosión alternante del Paleozoico de la Demanda o del Mesozoico en función de la tectónica. Si el primer planteamiento es correcto, la edad de los conglomerados sueltos coincidiría con la de las etapas frías del Mioceno Superior (Pontiense, postcabalgamiento), del Mioceno Medio (Vindoboniense) y Oligoceno (inferiores al Chatiense, para los más bajos).

\section{AGRADECIMIENTOS}

Al Instituto de Estudios Riojanos que subvenciona el Plan de Investigación «Movimientos recientes de la corteza terrestre en La Rioja y su influencia en los caracteres químico-térmicos del agua subterránea», para el que se ha hecho este estudio.

\section{BIBLIOGRAFIA}

Castiella, J., Sole, J., Villalobos, L., Del Valle, J., Ramirez, J. (1977). Mapa Geológico de España. 1:50.000. (253) CALAHORRA I.G.M.E., Hoja y Memoria.

Castiella, J., Del Valle, J. (1975). Mapa Geológico de España. 1:50.000 (204) LOGROÑO. I.G.M.E. Hoja y Memoria.

Cuenca, G. (1983). Nuevo yacimiento de vertebrados del Mioceno del borde meridional de la Cuenca del Ebro. Est. Geol. (39) 217-224.

Cuenca, G. (1985). Los roedores (Mammalia) del Mioceno Inferior de Autol. Inst. Est. Riojanos. $96 \mathrm{pp}$.

Durantez, D., Sole, J., Castiella, J., Villalobos, L., Ramirez, J., Rivas, P., Del Pan, T., Chacon, J (1982). Mapa Geológico de España. 1:50.000. (281) CERVERA DEL RIO ALHAMA. I.G.M.E. Hoja y Memoria.

Daams, R., Van Der Meulen, A.J. (1984). Paleoenvironmental and paleoclimatic interpretation of micromammal faunal sucessions in the upper Oligocene and Miocene of North Central Spain. Interim. coll. RCMNS Paleobiolog. Contr. Montpellier (14, 2)241-257. 


\section{PEREZ, PAVIA Y PEREDA}

I.G.M.E. (1971). Mapa Geológico de España. 1:200.000 (21) LOGROÑO hoja y Memoria.

Martinez-Salanova, J. (1987). Estudio paleontológico de los micromaníferos del Mioceno Inferior de Fuenmayor (La Rioja). Inst. Est. Riojanos. 99 pp.

Ortega, A., Perez-Lorente, F. (1984). El Terciario de la Depresión de Arnedo. Berceo (2) 99-113.

RiBA, O. (1955), . Sobre la edad de los conglomerados terciarios del borde Norte de las Sierras de la Demanda y de Cameros. Not. y Com. del I.G.M.E. (39) 39-50

Rrba, O. (1955). Sur le type de sedimentation du tertiaire continental de la partie ouest du bassin de l'Ebre Geol. Rund. (43) 263-271.

RibA, O. (1964). Estructura sedimentaria del Terciario continental de la Depresión del Ebro en su parte riojana y navarra. Comp. Red. XX $X^{e}$ Congr. Geol. Int. 127-138.

Sanchez-Lozano, R. (1894). Descripción física, geológica y minera de la provincia de Logroño. Mem. Com. Map. Geol. Esp. (1) 548 pp.

Schriel, W. (1930). Die Sierra de la Demanda und die Montes Obarenes. Abh. Ges. Wis. z. Gottingen, Math. Phys.

Sole Sabaris, L., Riba, O. (1952). Evolución del borde NE de la Meseta Española durante el Terciario. Comp. Rend. XIX Congr. Geoil, Int. Alger (XIII, 1e) 261-274. 